Off beam
$\begin{aligned} & \text { Cloudy horizon } \\ & \text { for Australian } \\ & \text { synchrotron } \\ & 0210\end{aligned}$

\title{
Biologists take tentative steps towards bespoke cancer drugs
} better tailored to an individual's genetic make-up or physiological condition.

The studies are focused on the anticancer drug Iressa. Last July, Japan became the first country to approve the therapy, after it sped through the country's new fast-track drugapproval system. Despite the 246 deaths so far ascribed to its side-effects, 28,000 Japanese lung-cancer patients are using Iressa, and the United States and Australia this month approved the drug.

Produced by Anglo-Swedish company AstraZeneca, Iressa is one of a revolutionary new generation of therapies based on an understanding of the specific gene mutations that cause individual cancers (see Nature 416, 470-474;2002). The drug is a tyrosine-kinase inhibitor, which blocks specific growthfactor receptors - proteins on the cell membrane that pass on signals commanding the cell to grow and are overactivated in cancer.

Despite hopes that this type of drug would cause side-effects in very few patients, 616 people receiving Iressa in Japan have developed severe reactions - mostly a pneumonia-like illness called interstitial lung disease (ILD). Nonetheless, health officials say that the drug works too well for it to be recalled given the lack of other options for treating the disease. The health ministry says that various hospitals throughout the country have reported reductions in tumour size in between onesixth and one-third of cases.

The two studies, which will begin shortly, are collaborations between universities and AstraZeneca. One will track the levels of certain blood proteins in different patients, and the other will examine the genetic differences between individuals called single-nucleotide polymorphisms (SNPs).

The SNPs project, led by Yusuke Nakamura of the University of Tokyo, will scour some

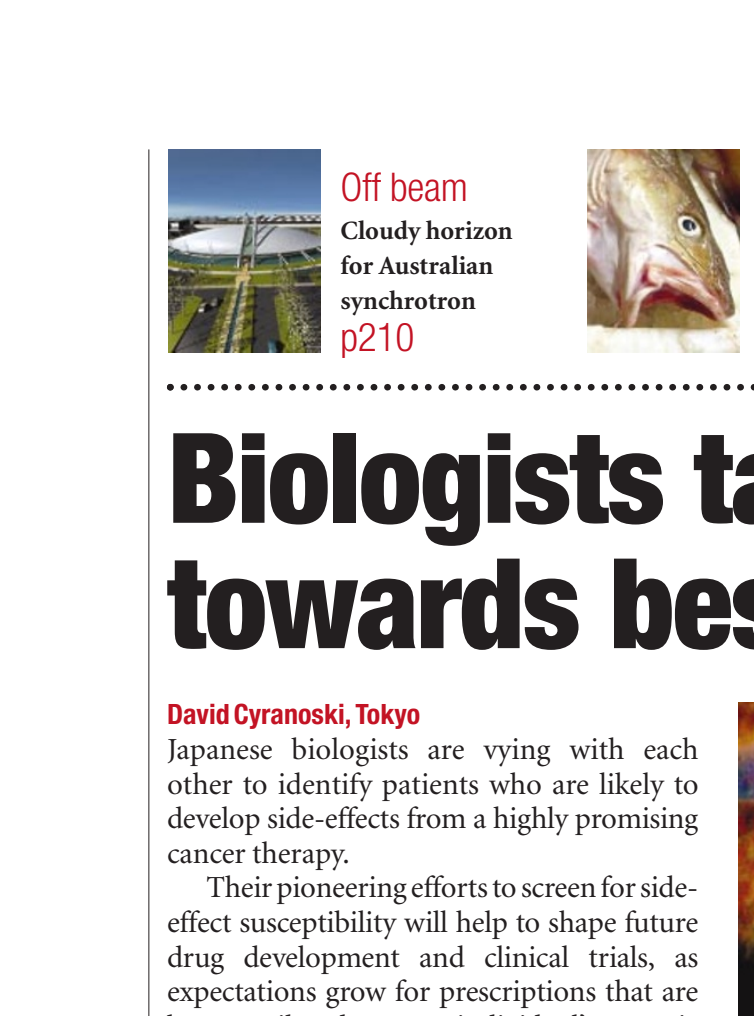

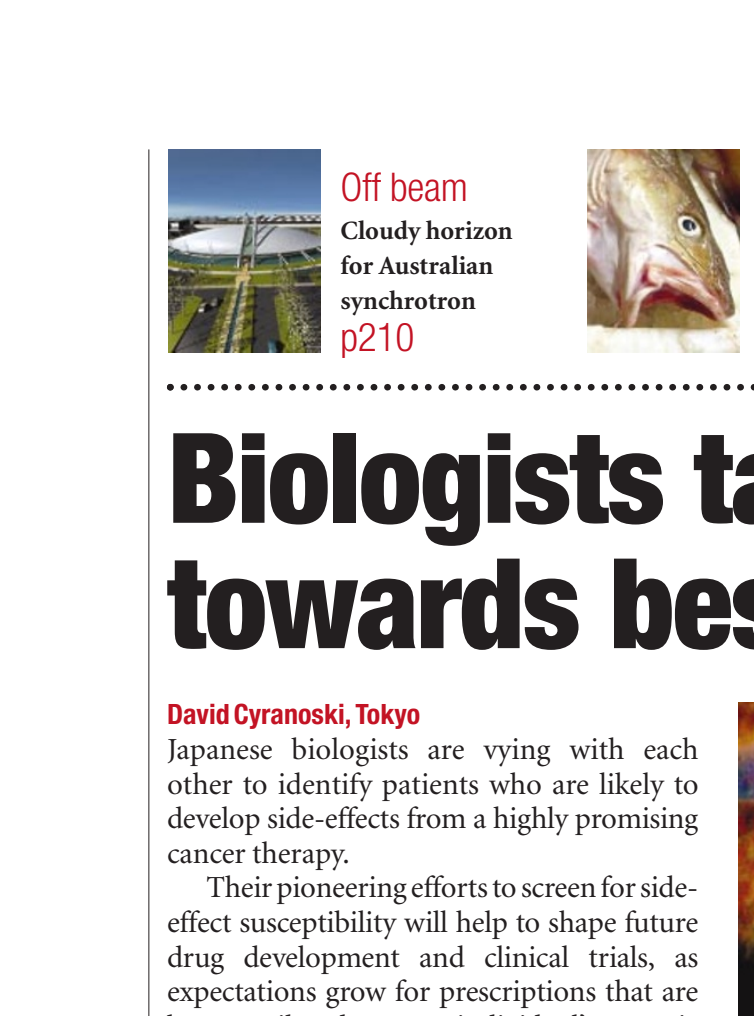

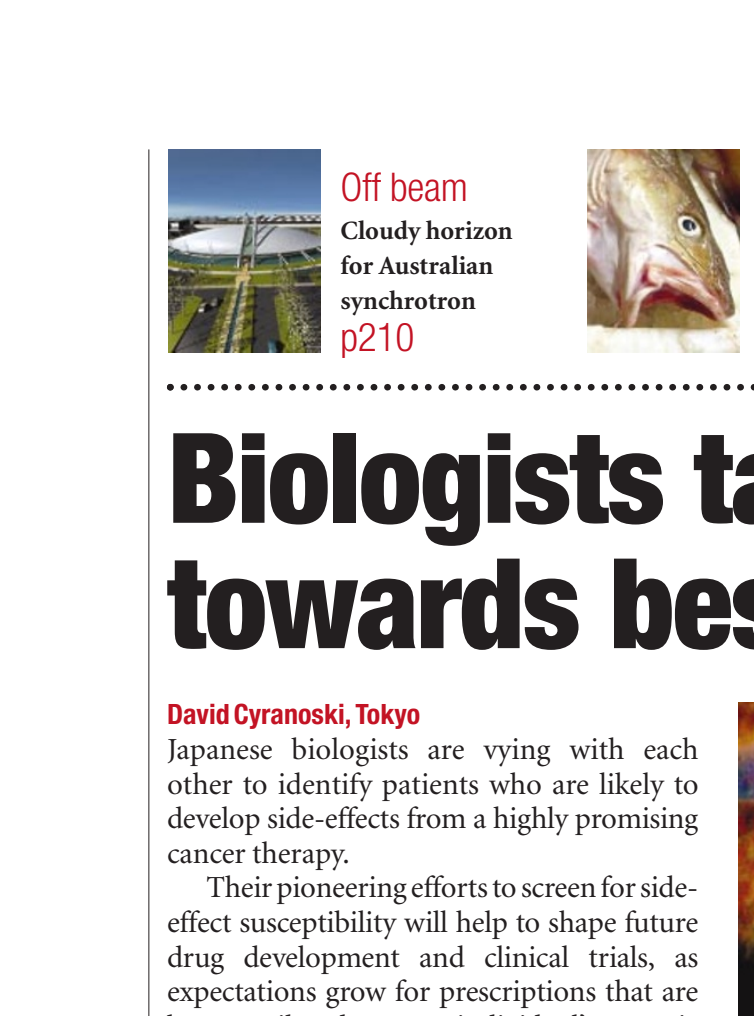

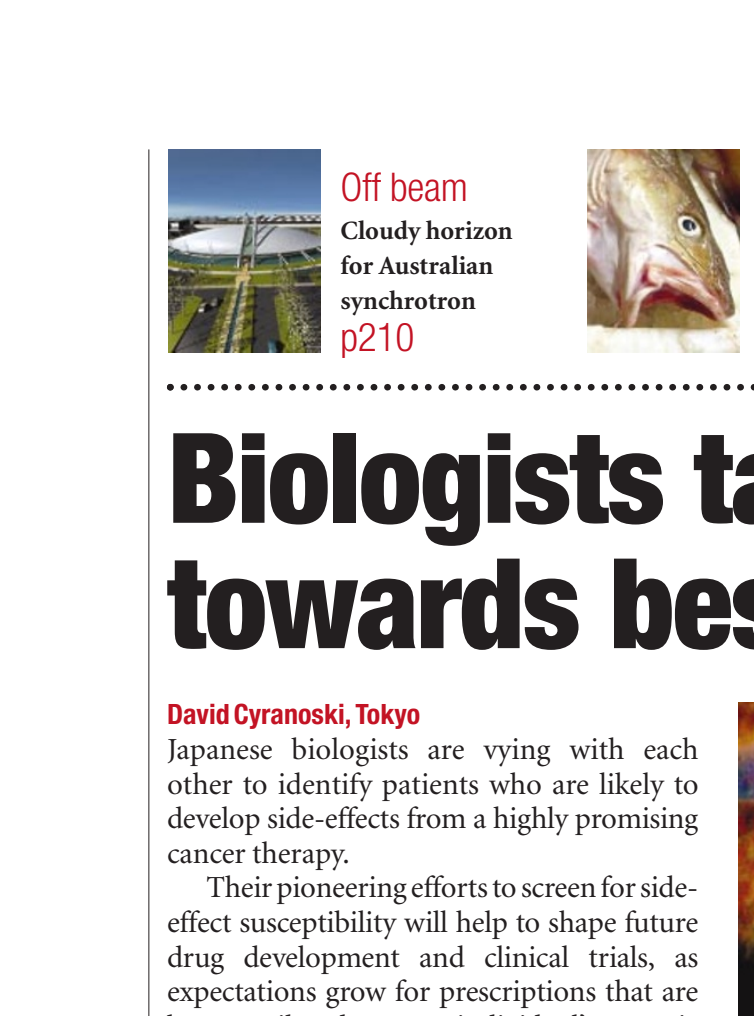

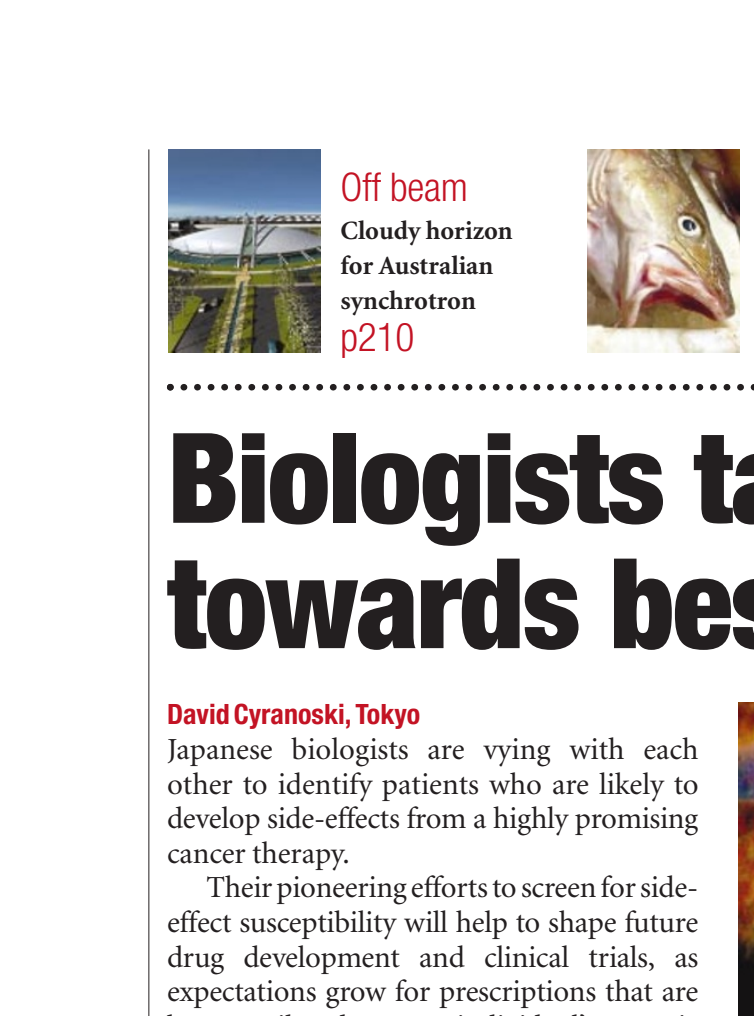

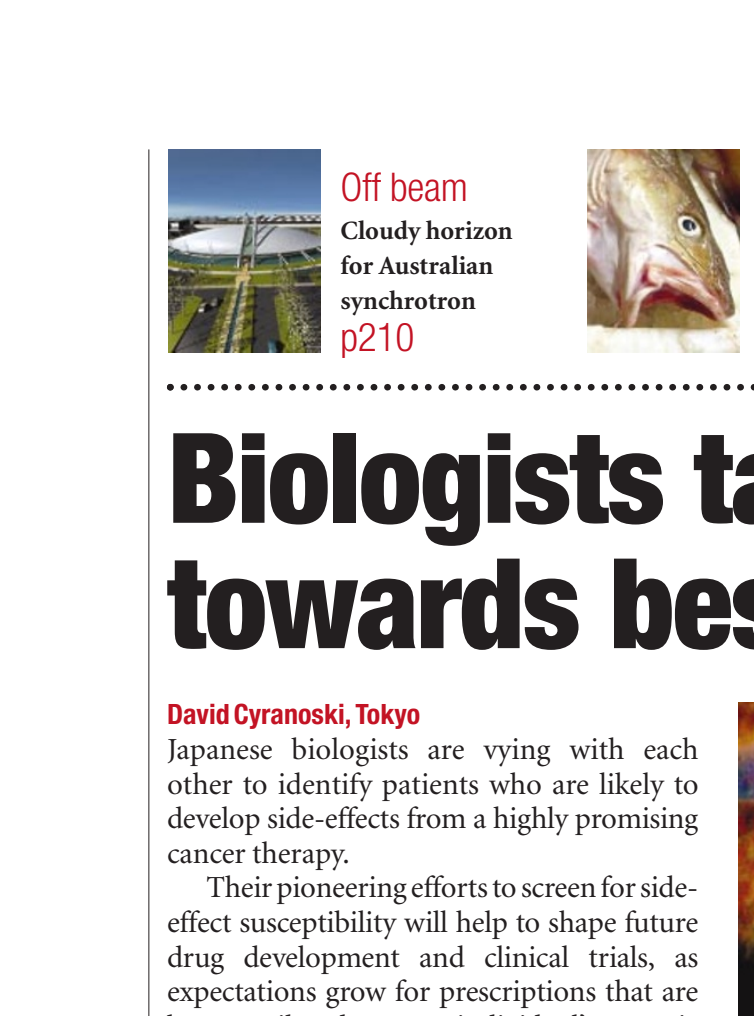

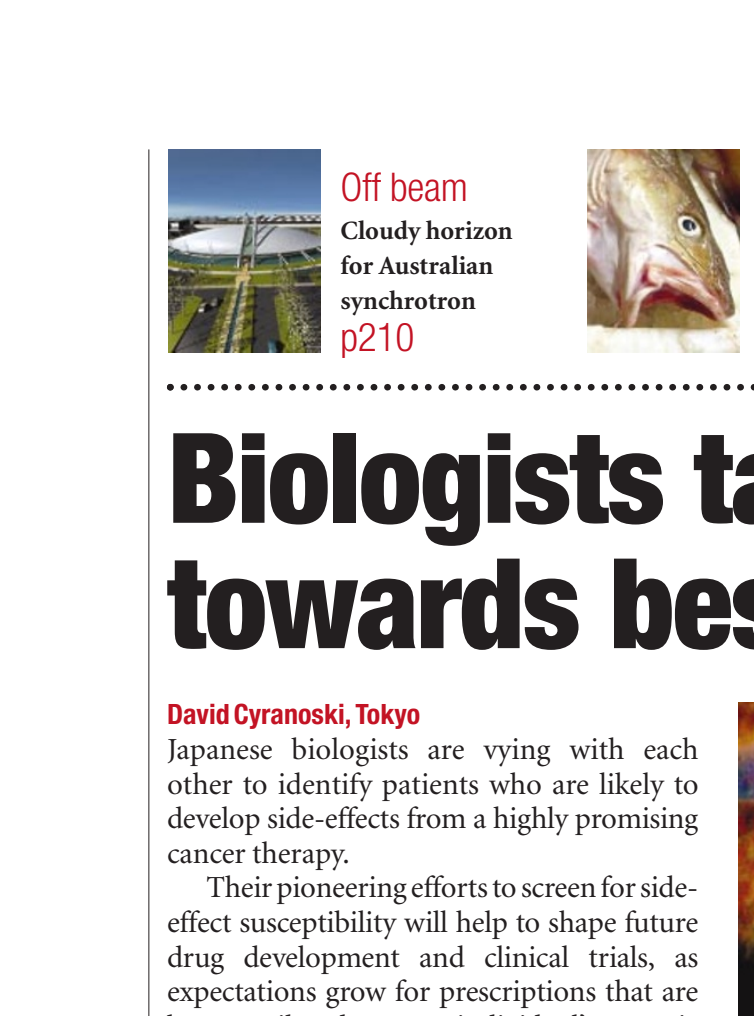

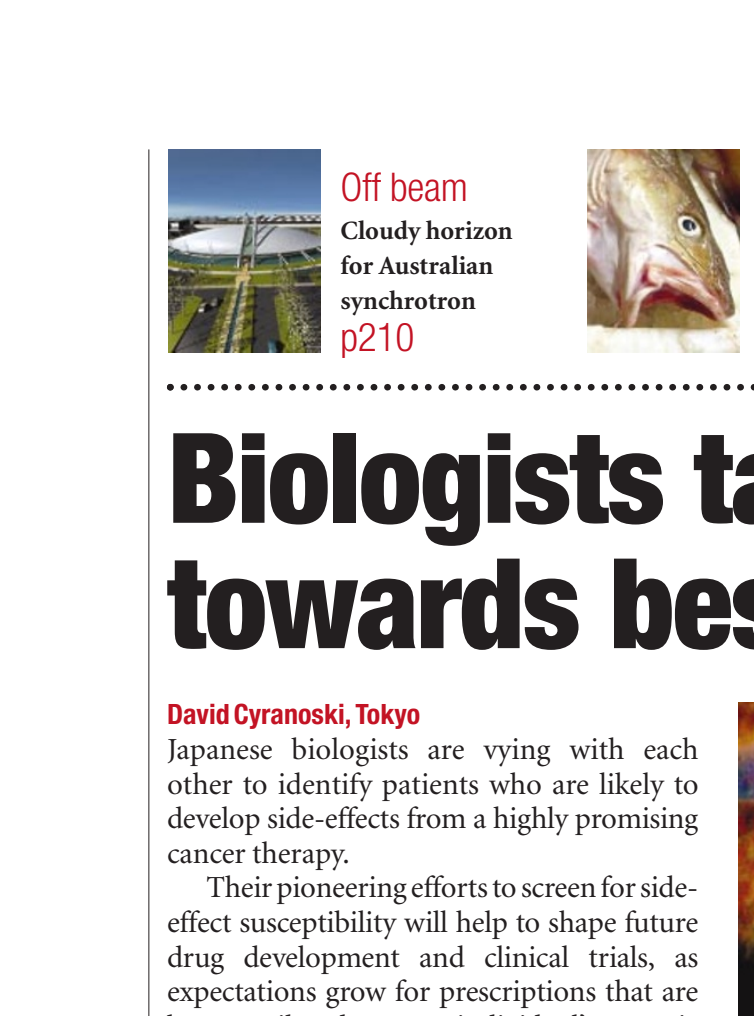

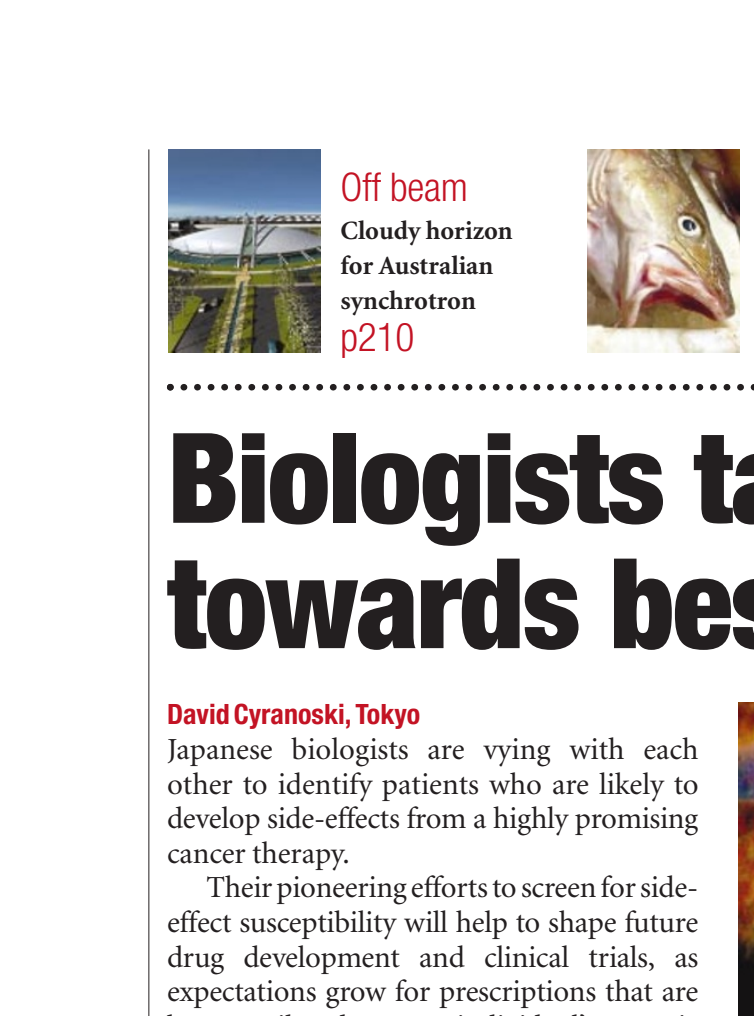
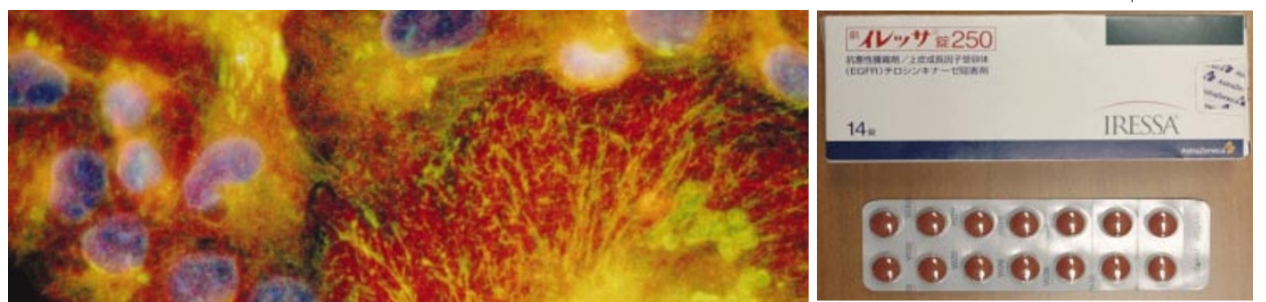

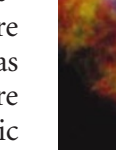

.
}

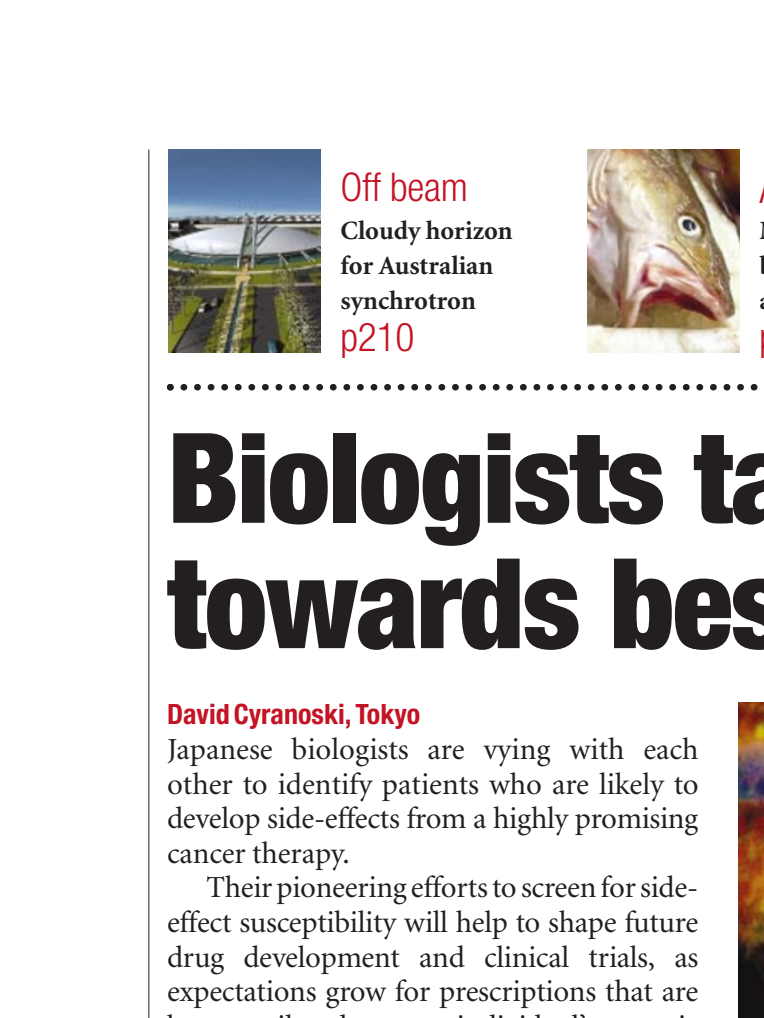

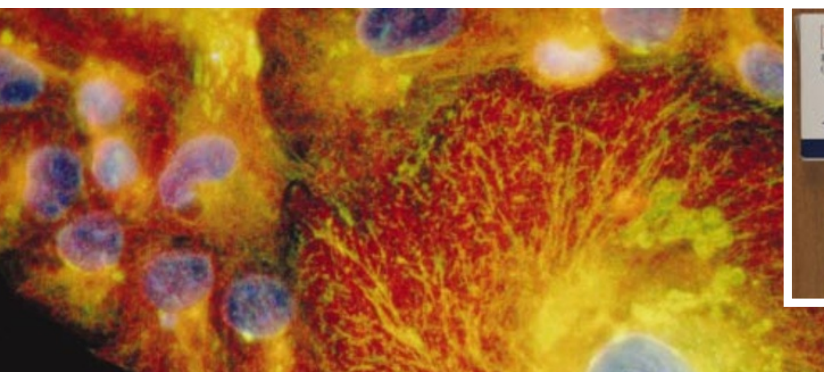
OQBOABO



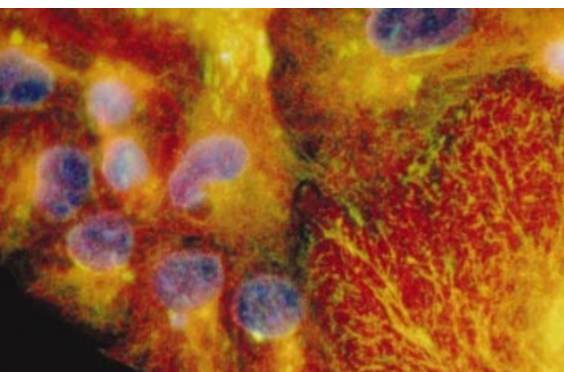

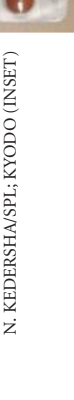

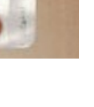

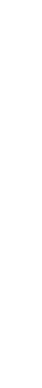

